\title{
Determination of Functional Outcomes of Displaced Olecranon Fracture Fixed with Intermedullary Partially Threaded Screw Augmented with Tension Band Wiring
}

\author{
MUHAMMAD KHIZER HAYAT MAKKI ${ }^{1}$, GHULAM QADIR KHAN ${ }^{2}$, ABDUL MUNAF SAUD ${ }^{3}$, SHAHID MEHMOOD ${ }^{4}$, \\ ZULFIQAR AHMED ${ }^{5}$, HAFIZ MUHAMMAD AHMAD QURESHI ${ }^{6}$ \\ ${ }^{1}$ Medical Officer, Bahawal Victoria Hospital /Civil (Sadiq Abbasi) Hospital, Bahawalpur. \\ ${ }^{2}$ Assistant Professor, Bahawal Victoria Hospital/ QAMC, Bahawalpur. \\ ${ }^{3}$ Assistant Professor of Orthopedic Surgery, Bahawal Victoria Hospital/Civil (Sadiq Abbasi) Hospital, Bahawalpur. \\ ${ }^{4}$ Assistant Professor of Orthopedic Surgery, Bahawal victoria Hospital, Bahawalpur. \\ ${ }^{5}$ Assistant Professor of orthopedic Surgery, Bahawal victoria Hospital/Civil (Sadiq Abbasi) Hospital, Bahawalpur \\ ${ }^{6}$ House Officer, Bahawal Victoria Hospital, Bahawalpur \\ Corresponding author: Dr Muhammad Khizer Hayat Makki, Email: makkikhizer@hotmail.com, Cell No:+923336142389
}

\begin{abstract}
Introduction: Orthopaedic injuries involving the olecranon are among the most often encountered in the emergency department.

Objective: To determine the functional outcomes of displaced olecranon fracture fixed with intermedullary partially threaded screw augmented with tension band wiring

Material and methods: This Descriptive/multicentric case series was conducted in Department of Orthopedics, Bahawal Victoria Hospital/Civil (Sadiq Abbasi) Hospital, Bahawalpur during June 2019 to June 2021. Patients who came through the emergency department and met the inclusion criteria were enrolled and given informed permission after clearance from the hospital's ethics committee. During general anesthesia or a biere's block, all patients received tension band wire and intramedullary screw fixation.

Results: Functional outcomes of displaced olecranon fracture fixed with intermedullary partially threaded screw augmented with tension band wiring was recorded as excellent in $57.39 \%(n=66), 32.17 \%(n=37)$ had good outcome, $10.44 \%(\mathrm{n}=12)$ had fair outcome.

Conclusion: We concluded that that functional outcomes of displaced olecranon fracture fixed with intermedullary partially threaded screw augmented with tension band wiring is good; however, validation is required through other local studies.

Key words: Olecranon fracture, Intramedullary partially threaded screw, Tension band wiring, and Campbell's posterolateral approach
\end{abstract}

\section{INTRODUCTION}

Olecranon fractures are one of the most common fractures observed in the emergency room. It is responsible for ten percent of all fractures of the upper extremities. 1 Olecranon fractures are most often caused by a direct strike during a fall or an indirect blow during a car collision following a severe triceps contraction. Elbow fractures are less common; however they can happen if the elbow is overextended. ${ }^{2}$ Olecranon is located beneath the skin, making it vulnerable to fractures. Olecranon fractures in young individuals following high energy trauma like a car accident and low energy trauma such a fall in the elderly. An Olecranon fracture usually occurs by itself, but it's important to check for any additional wounds or broken bones on the ipsilateral side as well. Fracture dislocation may occur as a result, changing the treatment strategy. ${ }^{3}$

In order to have a successful functional outcome, the articular surface must be anatomically restored, the elbow extensor mechanism repaired, joint stability and motion restored, and stiffness and other problems avoided. Olecranon fracture therapy now includes a variety of options. ${ }^{4}$

In general, the TBW principle states that when muscles contract, compressive and tensile pressures are generated on the affected bone. Whenever a bone is fractured, it causes a gap on the side of the bone that is receiving traction. With a TBW, the tensile forces are neutralized and compressed. This procedure ensures that the bone pieces are compressed dynamically and heal quickly. ${ }^{5}$ Complications might arise, the most prevalent of which include hardware prominence that needs to be removed, mobility loss and fixation loss.

Present study was conducted aimed to determine of functional outcomes of displaced olecranon fracture fixed with intermedullary partially threaded screw augmented with tension band wiring

\section{MATERIAL AND METHODS}

This Descriptive case series was conducted in Department of Orthopedics, Bahawalpur victoria Hospital/Civil (Sadiq Abbasi) Hospital, Bahawalpur during June 2019 to June 2021. The data was collected through non-probability consecutive sampling technique.

Inclusion Criteria

- Patients having age between 15-60 years of both genders.

- Patients having Transverse type of olecranon fracture.

Exclusion criteria:

- Patients having comminuted fractures of olecranon.

- Patients with avulsion fractures of olecranon.

- Patients with fracture dislocation.

Data Collection Procedure: Patients who came through the emergency department and met the inclusion criteria 
were enrolled and given informed permission after clearance from the hospital's ethics committee. General anesthesia or a biere's block was used to perform intermedullary partially threaded screw augmented with tension band wiring fixation on all of the patients. The anesthesiologist administered the anesthesia. Campbell's posterolateral technique was used to expose the olecranon. Senior consulting orthopedic surgeon did the operation. During the first day, the patient was asked to move his or her fingers by elevating the injured limb. After the third postoperative day, elbow movements were encouraged. After six weeks of treatment, the functional outcome was evaluated using the Broberg and Morrey grading method.

Motion, strength, stability, and pain are all included in this part. There was no pain, mild pain with exercise but no medicine was required, moderate pain with or after activity, severe pain at rest, continual medication was required, and the pain was disabled. To get the clinical and biomechanical evaluations, researchers used a hand goniometer to measure motion, the elbow's flexion/extension, and the forearm's pronation/supination. A torque dynamometer custom-made for this purpose was used to gauge the hand's grip strength. Varus-valgus instability was used to assign a grade to the overall stability of the system.

No instability was found; mild, if the patient perceives instability; moderate, if unequivocal instability is discovered; severe, if the physician detects and perceives noticeable varus or valgus laxity.

Data analysis: SPSS V-25 was used to examine all of the data. All quantitative factors, such as age and BMI, were given a mean and standard deviation. All qualitative factors, such as gender and functional result, were tallied using frequencies and percentages. Age, BMI, and gender were all used as effect modifiers in a post-stratification chisquare test. The significance level was set at P-values less than 0.05 .

\section{RESULTS}

A total of 115 cases fulfilling the selection criteria were enrolled to determine the functional outcome of tension band wiring combined with intramedullary screw fixation for treatment of fracture of olecranon. Age distribution shows that $80 \%(n=92)$ were between $15-40$ years of age whereas $20 \%(n=23)$ were between $41-60$ years of age, mean+SD was calculated as $31.75+9.50$ years.

Table 01: Determination of functional outcomes of displaced olecranon fracture fixed with intermedullary partially threaded screw augmented with tension band wiring $(n=115)$

\begin{tabular}{|l|l|l|}
\hline Functional outcome & No. of patients & $\%$ \\
\hline Excellent & 66 & 57.39 \\
\hline Good & 37 & 32.17 \\
\hline Fair & 12 & 10.44 \\
\hline Poor & 0 & 0 \\
\hline Total & 115 & 100 \\
\hline
\end{tabular}

Gender distribution shows that $78.26 \%(n=90)$ were male and $21.74 \%(n=25)$ were females. Mean Body mass index was calculated as $29.55+2.91$. Functional outcomes of displaced olecranon fracture fixed with intermedullary partially threaded screw augmented with tension band wiring was recorded as excellent in $57.39 \%$ $(n=66), 32.17 \%(n=37)$ had good outcome, $10.44 \%(n=12)$ had fair outcome.

Effect modifiers like age, BMI and gender were stratified and post-stratification chi-square test was applied. $P$-value $\leq 0.05$ was considered significant.

Table 02: Stratification for determination of functional outcomes of displaced olecranon fracture fixed with intermedullary partially threaded screw augmented with tension band wiring with regards to age $(n=115)$

\begin{tabular}{|c|c|c|c|}
\hline \multirow[b]{2}{*}{ Functional outcome } & \multicolumn{2}{|c|}{ Age(in years) } & \multirow[b]{2}{*}{$P$ value } \\
\hline & $15-40$ & $41-60$ & \\
\hline \multirow[b]{2}{*}{ Excellent $(\mathrm{n}=66)$} & 50 & 16 & \multirow[b]{2}{*}{0.19} \\
\hline & 42 & 7 & \\
\hline \multirow[b]{2}{*}{ Good(n=37) } & 32 & 5 & \multirow[b]{2}{*}{0.23} \\
\hline & 60 & 18 & \\
\hline \multirow[b]{2}{*}{ Fair $(n=12)$} & 10 & 2 & \multirow[b]{2}{*}{0.76} \\
\hline & 82 & 21 & \\
\hline
\end{tabular}

Table 03: Stratification for determination of functional outcomes of displaced olecranon fracture fixed with intermedullary partially threaded screw augmented with tension band wiring with regards to gender $(n=115)$

\begin{tabular}{|l|l|l|l|}
\hline Functional outcome & Gender & P value \\
\cline { 2 - 3 } & Male & Female & \\
\hline \multirow{2}{*}{ Excellent $(\mathrm{n}=66)$} & 51 & 15 & 0.77 \\
\cline { 2 - 3 } & 39 & 10 & \\
\hline \multirow{2}{*}{ Good $(\mathrm{n}=37)$} & 27 & 10 & 0.34 \\
\cline { 2 - 3 } & 63 & 15 & \\
\hline \multirow{2}{*}{ Fair $(\mathrm{n}=12)$} & 12 & 25 & 0 \\
& 78 & 0 & \\
\hline
\end{tabular}

Table No. 04: Stratification for determination of functional outcomes of displaced olecranon fracture fixed with intermedullary partially threaded screw augmented with tension band wiring with regards to $\mathrm{BMI}(\mathrm{n}=115)$

\begin{tabular}{|c|c|c|c|}
\hline \multirow[t]{2}{*}{ Functional outcome } & \multicolumn{2}{|l|}{$\mathrm{BMI}$} & \multirow[t]{2}{*}{$P$ value } \\
\hline & Upto 30 & $>30$ & \\
\hline \multirow[t]{2}{*}{ Excellent $(n=66)$} & 36 & 30 & \multirow[t]{2}{*}{0.78} \\
\hline & 28 & 21 & \\
\hline \multirow[t]{2}{*}{$\operatorname{Good}(\mathrm{n}=37)$} & 21 & 16 & \multirow[t]{2}{*}{0.87} \\
\hline & 43 & 35 & \\
\hline \multirow[t]{2}{*}{ Fair $(\mathrm{n}=12)$} & 7 & 5 & \multirow[t]{2}{*}{0.84} \\
\hline & 57 & 46 & \\
\hline
\end{tabular}

\section{DISCUSSION}

Among orthopaedic injuries observed in emergency rooms, olecranon fractures are a prevalent occurrence ${ }^{7-8}$. The articular surface and normal elbow function must be restored when they are dislocated through open reduction and internal fixation. Stability of the fixation, active elbow flexion and extension, and fracture union are all important.

Tension band wiring, intra fragmentary screws with or without wires, wires alone, and bone fragment excision with reattached triceps are a few of the ways that have been discussed in detail $^{9-10}$. In the treatment of olecranon fractures, the majority of research has focused on tension band wiring and intramedullary screw fixation ${ }^{11}$.

In Pakistan as well as worldwide in the last 5 years, however, the literature is lacking in assessing the functional outcome of combining these two operations for olecranon fracture. Research in this field can use the findings of this study as local references, and they will be helpful in the care of patients who have olecranon procedures because 
of the functional outcomes ${ }^{12}$. In our study, of 115 cases, $80 \%(n=92)$ were between $15-40$ years of age whereas $20 \%(n=23)$ were between $41-60$ years of age, mean+SD was calculated as $31.75+9.50$ years, $78.26 \%(n=90)$ were male and $21.74 \%(n=25)$ were females. Functional outcome of tension band wiring combined with intramedullary screw fixation for treatment of fracture of olecranon was recorded as excellent in $57.39 \%(n=66), 32.17 \%(n=37)$ had good outcome, $10.44 \%(n=12)$ had fair outcome. These findings are in agreement with a study, showing that excellent results were achieved in $60 \%$ patients, good results were achieved in $12 \%$ patients and fair results were achieved in $28 \%$ patients $^{6}$. There was no poor results non is good, however, validation is required through other local studies.

\section{CONCLUSION}

We concluded that the functional outcome of tension band wiring combined with intramedullary screw fixation for treatment of fracture of olecranon is good, however, validation is required through other local studies.

\section{REFERENCES}

1. Ashraf RA, Khan J, Mustafa MS, Ahmed R. Comparison of functional outcome in mayo elbow performance score in olecranon fractures after treatment with tension band wiring and locking compression plate. Pak Armed Forces Med J 2018;68(5):1256-9.

2. Kashif M, Hussain $\mathrm{H}$, Ahmed A, Akram R, Zaman A, Javed $\mathrm{S}$, et al. Functional outcome of tension band wiring with $\mathrm{k}$ wires for olecranon fractures. Professional Med $\mathrm{J}$. 2019;26(8):1256-60.
3. Hong CC, Han F, Decruz J, Pannirselvam V, Murphy D. Intramedullary compression device for proximal ulna fracture. Singapore Med J 2015;56(2):e17-20.

4. Cha SM, Shin HD. Fixation of the various coronal plane fracture fragments, including the entire coronoid process, in patients with Mayo type IIB olecranon fractures- four methods of fixation. Indian J Orthop 2019;53(2):224-31.

5. Nowotny J, Bischoff F, Ahlfeld T, Goronzy J, Tille E, Nimtschke $U$, et al. Biomechanical comparison of bi- and tricortical k-wire fixation in tension band wiring osteosynthsis. Eur J Med Res 2019;24:33.

6. Raju SM, Gaddagi RA. Cancellous screw with tension band wiring for fractures of the olecranon. J Clin Diagn Res 2013;7(2):339-41.

7. Ren Y, Qiao H, Wei Z, Lin W, Fan B, Liu J, et al. Efficacy and safety of tension band wiring versus plate fixation in olecranon fractures: a systematic review and meta- analysis. J Orthop Surg Res 2016;11:137.

8. Rouleau DM, Sandman $E$, van Riet $R$, Galatz LM. Management of fractures of the proximal ulna. J Am Acad Orthop Surg 2013;21(3):149-60.

9. Newman SD, Mauffrey C, Krikler S. Olecranon fractures. Injury 2009;40 (6):575-81.

10. Veillette CJ, Steinmann SP. Olecranon fractures. Orthop Clin North Am 2008;39(2):229-36.

11. Anderson ML, Larson AN, Merten SM, Steinmann SP. Congruent elbow plate fixation of olecranon fractures. J Orthop Trauma 2007;21(6):386-93.

12. Flinterman HJ, Doornberg JN, Guitton TG, Ring D, Goslings JC, Kloen P. Long-term outcome of displaced, transverse, noncomminuted olecranon fractures. Clin Orthop Relat Res 2014;472 (6):195 